\title{
Isolated cardiac involvement of extranodal rosai- dorfman disease: an atypical presentation
}

\begin{abstract}
Rosai-Dorfman disease, also known as sinus histiocytosis with massive lymphadenopathy, an uncommon systemic histio-proliferative disease, characterized by the overproduction of histiocytes, which accumulate in lymph nodes throughout the body. The extranodal cardiac involvement of this disease was never reported so far.

Clinical summary: $36 y$ year old presented with history of class 2/4 dyspnoea of 2year history with one episode of lymphocyte predominant pericardial effusion for which he had taken antitubercular therapy. Echocardiogram showed oval homogenous mass in the RVOT with severe PAH. CMR revealed infiltrating mass lesion in relation to the ventricular outflow tracts, the antero-superior walls of the left atrium and basal left ventricle, the anterior basal interventricular septum and the aorto-pulmonary window. Surgical excision of the tumour was done and histology showed of pin point hemorrhages showed spindle cells and fibrohistiocytic proliferation admixed with plasma cells lymphocytes \& polymorphs. Histocytes were present in small clusters and singly with abundant pale cytoplasm and nuclei with vesicular chromatin. Phagocytosed neutrophils and lymphocytes were present in some of these cells (Emperipolesis). These were suggestive of lymphoproliferative disease consistent with Rosai-Dorfman Disease.
\end{abstract}

Conclusion: secondary causes of pulmonary hypertension and atypical presentations of rare diseases should be kept in mind.

Keywords: cardiac rosai-dorfman disease
Volume 3 Issue 2 - 2015

\author{
Vijaya Chaitanya, Niraj Gupta \\ Department of Cardiology, Sri Jayadeva Institute of Cardiology,
} India

Correspondence: Vijaya Chaithanya, Sri Jayadeva Institute of Cardiology, Jayanagar 9th Block, Bannergatta Road, Bangalore, India pin-560069, Tel 917022160996, Email drvijaychaitanya@gmail.com

Received: September 16, 2014 | Published: August 14, 2015

\section{Introduction}

Rosai-Dorfman disease, also known as sinus histiocytosis with massive lymphadenopathy, an uncommon systemic histio-proliferative disease, characterized by the overproduction of histiocytes, which accumulate in lymph nodes throughout the body. This condition was first described by Robb-Smith in 1947 in children and was termed as Giant Cell Sinus Reticulosis and recognised as distinct clinical -pathological entity in 1969. Lymphadenopathy of the neck is the commonest site of histiocytes accumulation, although accumulation outside of lymph nodes may also occur. The most common sites of accumulation outside of the lymph nodes are skin, upper respiratory tract, bone and the sinuses. This condition has been named after Ronald F Dorfman \& Juan Rosai. ${ }^{1}$ The disease is often benign and self-limited; however, occasionally it is highly aggressive and life threatening. ${ }^{1,2}$

\section{Clinical summary}

A 36years old male presented to our institute with history of atypical chest pain and breathlessness on exertion class II for past 2years. He had past history of pericardial effusion 3years back for which he took full course of ATT. General physical examination was unremarkable, although cardiovascular examination revealed loud P2 component of second heart sound and grade III/VI ejection systolic murmur over the pulmonary area. Electrocardiogram revealed RAD of QRS complex, incomplete RBBB \& RVH. X-ray chest PA view had prominent right ventricular outflow tract with no evidence of hilar or paratracheal lymphadenopathy (Figure 1).

Transthoracic echocardiography showed oval homogenous mass $2.6 \times 1.9 \mathrm{~cm}$ attached to lateral wall of MPA, protruding into the lumen, resulting in obstruction with peak gradient of $58 \mathrm{mmHg}, \mathrm{RV}$ pressure overload with severe PAH, LVH with increased echogenicity. Cardiac
MRI revealed (Figure 2-5) infiltrating mass lesion in relation to the ventricular outflow tracts, the antero-superior walls of the left atrium and basal left ventricle, the anterior basal interventricular septum and the aortopulmonary window. Circumferential thickening of the ascending aorta, aortic arch and the proximal descending aortic walls, encasement of the proximal coronary arteries and involvement of the pericardium and the pericardial fat was present.

Subsequently endovascular biopsy from RV was done. Mason's Trichomestain was positive for fibrous \& collagenous tissue, whereas Mucicarmine stain was negative for myxoma. Due to RVOT obstruction surgical debulking was done with RVOT patch enlargement from commercial pericardium via median sternotomy with cardiopulmonary bypass. Histopathology examination of multilobulated greyish white $3 \times 2.5 \times 1 \mathrm{~cm}$ biopsy material with areas of pin point haemorrhages showed spindle cells and fibro-histiocytic proliferation admixed with plasma cells lymphocytes \& polymorphs. Histiocytes were present in small clusters and singly with abundant pale cytoplasm and nuclei with vesicular chromatin. Phagocytosed neutrophils and lymphocytes were present in some of these cells (Emperipolesis) (Figure 6).

These were suggestive of lymphoproliferative disease consistent with Rosai-Dorfman Disease. Post operative echocardiography revealed small residual mass in RVOT with gradient of $10 \mathrm{mmHg}$. Pre-discharge contrast enhanced CT scan of thorax \& abdomen showed a residual infiltrating mass lesion in relation to the RVOT \& no lymphadenopathy. Intrapulmonary component of the mass was not visualised. The lungs, abdominal\& pelvic structures and the bone under view showed no obvious abnormality. Follow up at 1 month patient was asymptomatic \& echocardiography demonstrated small residual mass in RVOT with gradient of $12 \mathrm{mmHg}$. He is on low dose steroids and is doing well. 


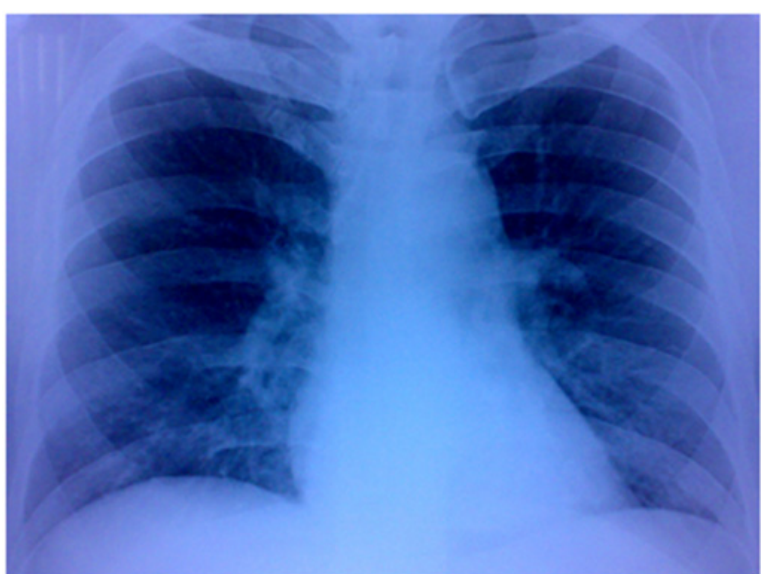

Figure I X-ray chest PA view had prominent right ventricular outflow tract with no evidence of hilar or paratracheal lymphadenopathy.

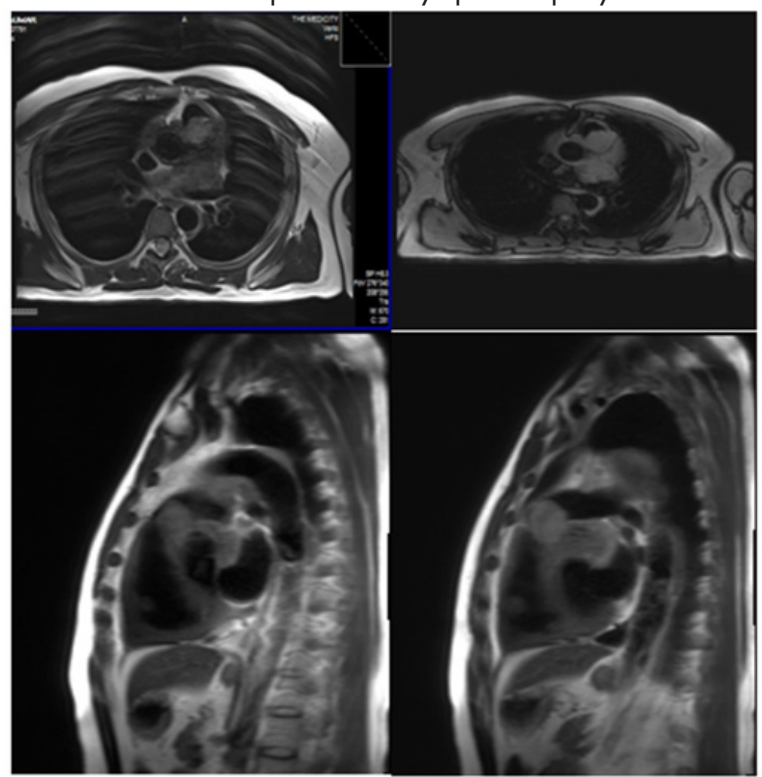

Figure 2-5 Cardiac MRI revealed infiltrating mass lesion in relation to the ventricular outflow tracts.

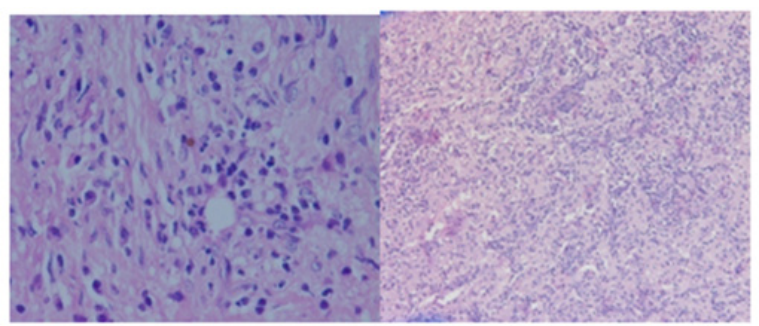

Figure 6 Phagocytosed neutrophils and lymphocytes were present in some of these cells Emperipolesis.

\section{Discussion}

Rosai-Dorfman Disease usually present in first or second decade of life with extranodal nodal involvement in about $25-43 \%$ of cases. Typical presentation is massive, painless, $\mathrm{B} / \mathrm{L}$ matted cervical lymphadenopathy with fever, leukocytosis, raised ESR, polyclonal hypergammaglobulinemia ${ }^{2}$. Autoimmune hemolysis can also occur. Our patient presented in fourth decade of life, no lymphadenopathy, no history of fever, normal ESR \& normal serum gamma globulin levels. Cardiac involvement of RDD was never reported in any literature making it's the first.

Exact pathogenesis of the disease is still not known. Theories implicating an infectious cause and immunodeficiency state have been suggested. By in situ hybridization, the presence of human herpes virus 6genome was demonstrated. Relationships with Klebsiella, EpsteinBarr virus, Brucella, or Cytomegalovirus were also suggested. It may be because of stimulation of monocytes / macrophages via M-GSF leading to immune suppressive macrophages. ${ }^{3,4}$

In regard to its natural behaviour, soft tissue Rosai-Dorfman Disease has been described as a slow growing process. ${ }^{5}$ Recurrence is common in soft tissue without lymph node involvement after local excision. Whenever feasible, the excision should be performed with wide surgical margins since chemotherapy has not proven useful. ${ }^{6}$ Surgery is usually reserved for obstructive or compressive symptoms. Death is because of extensive disease affecting vital organs, amyloidosis or cytotoxic toxicity. Our patient was submitted to a surgical intervention in which the excision was made to relieve obstructive symptoms followed by low dose steroid therapy.

Differential diagnosis includes non specific sinus hyperplasia, Langerhan's cell histiocytosis, metastatic malignant melanoma and lymphoma. Histologically, Rosai-Dorfman Disease and Hodgkin's lymphoma can present certain overlapping features, the latter occasionally presenting an inflammatory appearance and large neoplastic cells were resembling large histiocytes. However, these two diseases can easily be distinguished by identification of emperipolesis in Rosai-Dorfman Disease, identification of neoplastic Reed-Stenberg cells in Hodgkin's lymphoma. The present case highlights the importance of including extranodal Rosai-Dorfman Disease in the differential diagnosis of cardiac mass because this case had involvement of only cardiac structures and great vessels.

\section{Acknowledgments}

None.

\section{Conflicts of interest}

Author declares there are no conflicts of interest.

\section{Funding}

None.

\section{References}

1. Rosai J, Dorfman RF. Sinus histiocytosis with massive lymphadenopathy. A newly recognized benign clinicopathological entity. Arch Pathol. 1969;87(1):63-70.

2. Foucar E, Rosai J, Dorfman R. Sinus histiocytosis with massive lymphadenopathy (Rosai-Dorfman disease): review of the entity. Semin Diagn Pathol. 1990;7(1):19-73.

3. Kumar B, Karki S, Paudyal P. Diagnosis of sinus histiocytosis with massive lymphadenopathy (Rosai-Dorfman disease) by fine needle aspiration cytology. Diagn Cytopathol. 2008;36(10):691-695.

4. LampertF,LennertK. Sinus histiocytosis with massive lymphadenopathy: Fifteen new cases. Cancer. 1976;37(2):783-789.

5. Veinot JP, Eidus L, Jabi M. Soft tissue Rosai Dorfman disease mimicking inflammatory pseudotumor: a diagnostic pitfall. Pathology. 1998;30(1):14-16.

6. Montgomery EA, Meis JM, Frizzera G. Rosai-Dorfman disease of soft tissue. Am J Surg Pathol . 1992;16(2):122-129. 